\title{
„Open and Shut" für Gäste in Molybdän- Sauerstoff-Riesenkugeln, -körben und -rädern mit dem Pentagon als gemeinsamem Strukturelement
}

Achim Müller,* Sebastian Polarz, Samar K. Das, Erich Krickemeyer, Hartmut Bögge, Marc Schmidtmann und Björn Hauptfleisch

In memoriam Frank Hellweg

Die Synthese sehr großer molekularer Behälter ${ }^{[1]}$ oder Wirtsysteme, die problemorientiert handhabbar sind, stellt eine große Herausforderung dar. Wir berichten hier über die Bildung eines molekularen Riesenkorbes, der durch „Öffnung“ einer Molybdän-Sauerstoff-Riesenkugel („Keplerat" $\left.^{\text {" }}\right)^{[2]}$ entsteht, und über die Option, den Inhalt der entsprechenden Kugeln variabel und gesteuert auszutauschen. Die früher von uns geäußerte Prognose, daß sich aufbauend auf der Isolierung der ersten molekularen Keplerat-Riesenkugeln ${ }^{[2]}$ - eine neuartige Supramolekulare Chemie, also eine regelrechte Keplerat-Chemie entwickeln würde, ${ }^{[2]}$ ist damit bestätigt worden. In diesem Kontext ist eine Art Pythagoräischer Harmonie ${ }^{[3]}$ von besonderem Interesse, da das Krümmungen bewirkende Strukturelement für kugelund ringförmige Riesencluster, für die eine gemeinsame Formel $\left\{\mathrm{Mo}_{11}\right\}_{n}$ angegeben werden kann, dasselbe ist.

Die Verbindung 1 mit dem erwähnten molekularen Riesenkorb 1a als Anion, die zum Verständnis der unten angesprochenen Austauschreaktionen von Liganden in kugel-

\footnotetext{
*] Prof. Dr. A. Müller, Dipl.-Chem. S. Polarz, Dr. S. K. Das,

E. Krickemeyer, Dr. H. Bögge, M. Schmidtmann,

Dipl.-Chem. B. Hauptfleisch

Lehrstuhl für Anorganische Chemie I

Fakultät für Chemie der Universität

Postfach 100 131, D-33501 Bielefeld

Fax: (+49)521-106-6003

E-mail: a.mueller@uni-bielefeld.de
} 
förmigen Keplerat-Clustern offensichtlich von Bedeutung ist, kristallisiert beim längeren Stehen an der Luft aus Lösungen aus, die das Keplerat-Anion $\mathbf{2 a}^{[2]}$ enthalten (Abbildung 1). (1 konnte bisher allerdings nicht nebenproduktfrei isoliert werden.) Aus frisch hergestellten Lösungen von 2 lassen sich

$\mathrm{Na}_{46}\left[\mathrm{Mo}_{66}^{\mathrm{VI}} \mathrm{Mo}_{50}^{\mathrm{V}} \mathrm{O}_{331}\left(\mathrm{CH}_{3} \mathrm{COO}\right)_{30}\left(\mathrm{H}_{2} \mathrm{O}\right)_{56}\right] \cdot$ ca. $300 \mathrm{H}_{2} \mathrm{O}$ ca. $10 \mathrm{CH}_{3} \mathrm{COONH}_{4} \quad 2$

a)
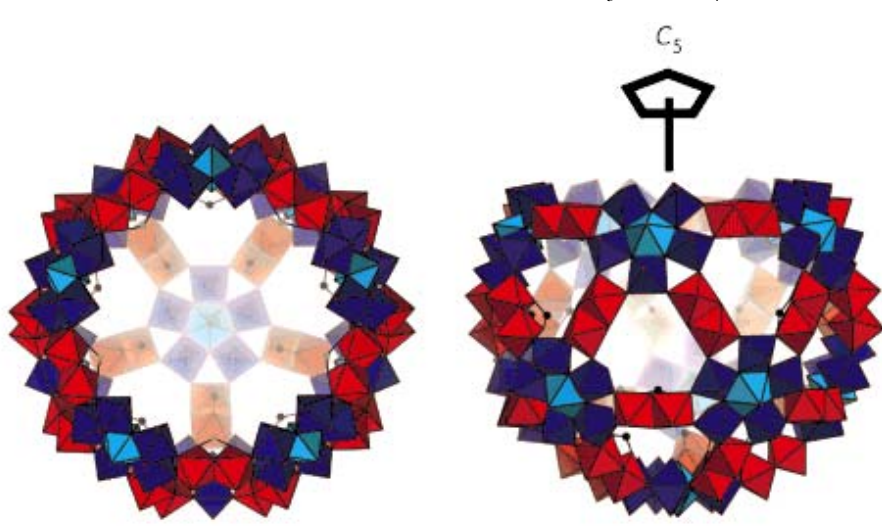

b)

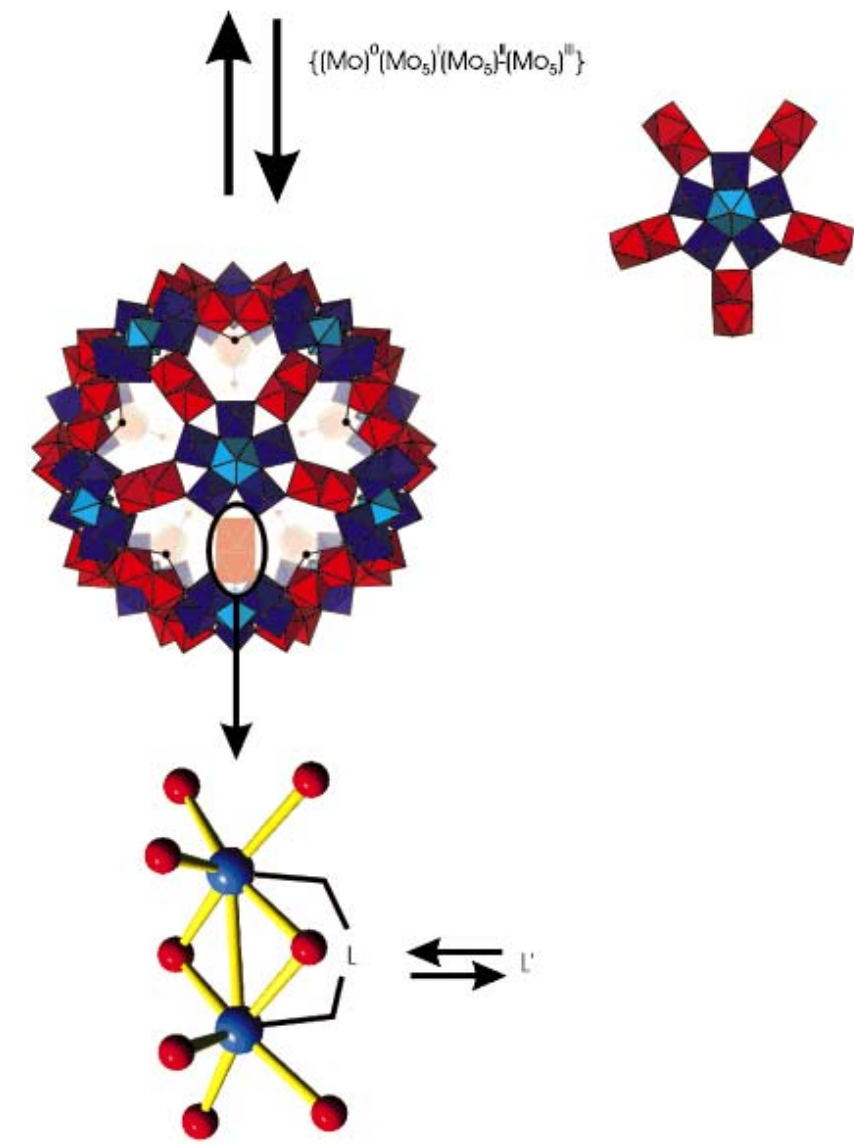

Abbildung 1. Struktur von a) $\mathbf{1 a}$ (Blick in Richtung der $C_{5}$-Achse und senkrecht dazu) und b) 2 a mit einer separat hervorgehobenen $\left\{\mathrm{Mo}_{2}^{\mathrm{V}} \mathrm{L}\right\}$-Einheit mit angedeuteter Position, an der die Ligandenaustauschreaktionen stattfinden. Das $\left\{\mathrm{Mo}_{16}\right\}$-Fragment (,Deckel“ des Korbes 1a) ist als Strukturmotiv separat dargestellt (\{(Mo)Mo $\left.\mathrm{Mo}_{5}\right\}$ Pentagone: blau; $\left\{\mathrm{Mo}_{2}^{\mathrm{V}}\right\}$-Fragmente: rot). nach Zugabe von koordinationsfähigen Anionen (z.B. Perchlorat-, Sulfat-, Sulfit-, Phosphat-, Hypophosphit- oder Phenylphosphonationen) Verbindungen mit entsprechend substituierten Keplerat-Clustern, also identischen Kugelschalen, in reiner Form und relativ hohen Ausbeuten isolieren. In den resultierenden Verbindungen sind die ursprünglich an den $\left\{\mathrm{Mo}_{2}^{\mathrm{V}} \mathrm{O}_{4}{ }^{2+}\right\} \equiv\left\{\mathrm{Mo}_{2}^{\mathrm{V}}\right\}$-Gruppen koordinierenden $\mathrm{CH}_{3} \mathrm{COO}^{-}$-Liganden von $2 \mathbf{a}^{[2]}$ entweder ganz (wie im Falle des Phosphats und Hypophosphits) oder partiell ausgetauscht (wie im Falle des Sulfats). Hier werden die Verbindungen 3 und 4 beschrieben. ${ }^{[4]}$

$\left(\mathrm{NH}_{4}\right)_{42}\left[\left\{\left(\mathrm{Mo}^{\mathrm{VI}}\right) \mathrm{Mo}_{5}^{\mathrm{VI}} \mathrm{O}_{21}\left(\mathrm{H}_{2} \mathrm{O}\right)_{6}\right\}_{12}\left\{\mathrm{Mo}_{2}^{\mathrm{V}} \mathrm{O}_{4}\left(\mathrm{H}_{2} \mathrm{PO}_{2}\right)\right\}_{30}\right] \cdot$ ca. $300 \mathrm{H}_{2} \mathrm{O} \quad 3$

$\left(\mathrm{NH}_{4}\right)_{42}\left[\left\{\left(\mathrm{Mo}^{\mathrm{VI}}\right) \mathrm{Mo}_{5}^{\mathrm{VI}} \mathrm{O}_{21}\left(\mathrm{H}_{2} \mathrm{O}\right)_{6}\right\}_{12}\left\{\mathrm{Mo}_{2}^{\mathrm{V}} \mathrm{O}_{4}\left(\mathrm{HSO}_{4}\right)\right\}_{18}\left\{\mathrm{Mo}_{2}^{\mathrm{V}}\right.\right.$

$\left.\left.\mathrm{O}_{4}\left(\mathrm{CH}_{3} \mathrm{COO}\right)\right\}_{12}\right] \cdot \mathrm{ca} .300 \mathrm{H}_{2} \mathrm{O} \quad 4$

$\mathbf{1}, \mathbf{3}$ und $\mathbf{4}$ wurden elementaranalytisch (einschließlich cerimetrischer Titration zur Bestimmung der Zahl der $\mathrm{Mo}^{\mathrm{V}}$-Zentren), thermogravimetrisch (zur Bestimmung des Kristallwassergehaltes), spektroskopisch (IR-, Raman-, UV/Vis-Spektroskopie) und röntgenstrukturanalytisch ${ }^{[5]}$ unter Einbeziehung von Bindungsvalenzsummen(BVS)-Berechnungen ${ }^{[6]}$ (zur Ermittlung der Lage der $\mathrm{H}_{2} \mathrm{O}$-Moleküle sowie der Unterscheidung der $\mathrm{Mo}^{\mathrm{VI}}$ - und $\mathrm{Mo}^{\mathrm{V}}$-Zentren) charakterisiert.

Die Verbindung 1 mit dem korbförmigen Anion kristallisiert in der Raumgruppe $P m n 2_{1} .^{[5]} \mathbf{3}$ und 4 sind isostrukturell bzw. pseudoisostrukturell zu den entsprechenden Verbindungen mit Riesenkugeln des Typs $\left\{\mathrm{Mo}_{132}\right\}$, die Acetatliganden ${ }^{[2]}$ (Raumgruppe $F m \overline{3}$ ) bzw. Formiatliganden ${ }^{[7]}$ (Raumgruppe $R \overline{3}$ ) an den $\left\{\mathrm{Mo}_{2}^{\mathrm{V}}\right\}$-Gruppen enthalten. Die Struktur des korbförmigen Riesenclusters 1a läßt sich aus der Riesenkugel 2a $\mathbf{a}^{[2]} \quad$ (Abbildung 1) $\quad\left(\left\{\mathrm{Mo}_{11}\right\}_{12} \equiv\right.$ $\left.\left\{(\mathrm{Mo})^{0}\left(\mathrm{Mo}_{5}\right)^{\mathrm{I}}\left(\left\{\mathrm{Mo}_{2}^{\mathrm{V}}\right\}_{5 / 2}\right)^{\mathrm{III}}\right\}_{12}\right)$ formal ,durch Entfernung" eines $\left\{(\mathrm{Mo})^{0}\left(\mathrm{Mo}_{5}\right)^{\mathrm{I}}\left\{\left(\mathrm{Mo}^{\mathrm{V}}\right)^{\mathrm{II}}-\left(\mathrm{Mo}^{\mathrm{V}}\right)^{\mathrm{III}}\right\}_{5}\right\}$-Fragmentes/Motivs (Abbildung 1) ableiten. Hierbei ist allerdings die Verteilung der Acetatliganden, die sich am „Korbrand“ - auch unter Einbeziehung der Koordination an die $\left\{(\mathrm{Mo}) \mathrm{Mo}_{5}\right\}$-Einheiten - konzentrieren, etwas verändert. Auffallend ist, daß der relative Anteil der $\mathrm{Mo}^{\mathrm{V}}$-Zentren in $\mathbf{1}$ a geringer ist als in $\mathbf{2 a}$, so daß der Reaktion (bezogen auf $\mathbf{1}$ a und $\mathbf{2 a}$ ) formal eine partielle Oxidation zugrunde liegt.

Das nach „Öffnung“ der molekularen Riesenkugel 2 a entstehende Produkt 1 mit dem korbförmigen Anion 1a ist in $\mathrm{H}_{2} \mathrm{O}$ erheblich besser löslich als 2, dessen Anion 2a auf der Kugeloberfläche vorwiegend terminale Sauerstoffliganden enthält. Der Grund für den Löslichkeitsunterschied sollte der leichte Zugang des Lösungsmittels zum hydrophilen Innenraum von 1a sein, der eine große Zahl zu terminalen $\mathrm{O}$-Atomen trans-ständiger $\mathrm{H}_{2} \mathrm{O}$-Liganden aufweist. Die Kristallisation von 1 erfolgt daher erst, nachdem sich das Gleichgewicht weitgehend in Richtung des Korbes verschoben hat, wobei man anfangs von einem relativ großen Lösungsvolumen ausgehen muß, damit der weniger lösliche kugelför- 
mige Cluster 2a lange genug in Lösung bleibt. Arbeitet man in Gegenwart einer hohen Natriumacetat-Konzentration (bewirkt Aussalzen durch Zerstörung der Hydratschale) oder auch in Gegenwart von $\mathrm{NH}_{4}{ }^{+}$-Ionen, die eine geringere Löslichkeit des relevanten Salzes bewirken, wird das Syntheseziel (1) nicht erreicht, und 2 fällt aus.

Um den bemerkenswerten Gast-/Ligandenaustausch bei der Reaktion von $2 \mathbf{a}$ zu 3a oder $4 \mathbf{a}$ zu verstehen, sollte folgendes berücksichtigt werden:

1) Der Ligandenaustausch sollte nicht durch die auf den $C_{3}$-Achsen befindlichen "molekularen Fenster" (zumindest solange es sich hier um starre $\left\{\mathrm{Mo}_{9} \mathrm{O}_{9}\right\}$-Ringe handelt) von $\mathbf{2 a}$ erfolgen, da diese für die genannten negativ geladenen Ionen zu eng dimensioniert sind.

2) Die Isolierung von $\mathbf{3}$ und $\mathbf{4}$ erfolgt zu einem Zeitpunkt, an dem in der Reaktionslösung noch ein erheblicher Anteil der kugelförmigen Anionen vorhanden ist.

3) Aus (1) folgt, daß entweder die ,an die Kugel 2a herantretenden“ Liganden eine „Öffnung“ bewirken, oder daß in Lösung ein Gleichgewicht zwischen einem entsprechenden korbförmigen Gebilde und der „geschlossenen“ Kugel vorliegt - mit der Möglichkeit des direkten Ligandenaustausches. Im letzteren Fall kann man zwanglos auch die leicht erfolgende Kristallisation der Verbindungen $\mathbf{3}$ und $\mathbf{4}$ (d.h. nach Ligandenaustausch und Einstellung eines neuen Gleichgewichtes) mit den entsprechenden Cluster-Kugeln 3a und 4 a verstehen, da diese aufgrund der relativ zu 1a geringeren Hydrophilie der Oberfläche der Kugel schwerer löslich sind als die entsprechenden, sich im Gleichgewicht befindenden Korbspezies. Für das Vorliegen des Gleichgewichtes spricht auch, daß aus einer Lösung, die ausschließlich mit der Korbverbindung 1 hergestellt wurde, das kugelförmige Clusteranion 2a (entsprechend 2) isoliert werden kann (das Umgekehrte gilt übrigens gleichermaßen). Bei diesen Überlegungen kann allerdings nicht davon ausgegangen werden, daß das $\left\{\mathrm{Mo}_{16}\right\}$-Motiv in Lösung als relativ stabile Einheit vorliegt.

Die Tatsache, daß beim Übergang Kugel $\rightarrow$ Korb ein Fragment des Typs $\left\{\left(\mathrm{Mo}_{11}\right) \mathrm{Mo}_{5}\right\}$, abgestoßen“ wird, regt $\mathrm{zu}$ Überlegungen an bezüglich der Relevanz von pentagonalen Baugruppen für eine relevante Strukturchemie. In Abbildung 1, die die Struktur von 2a mit Blick in Richtung der fünfzähligen Achse zeigt, ist das sternförmige Grundmotiv $\left\{(\mathrm{Mo})^{0}\left(\mathrm{Mo}_{5}\right)^{\mathrm{I}}\left(\mathrm{Mo}_{5}\right)^{\mathrm{II}}-\left(\mathrm{Mo}_{5}\right)^{\mathrm{II}}\right\}$ (vgl. auch Abbildung $\left.2 \mathrm{~b}\right)$, das also formal mehrere (Motiv-),,Generationen“ aufweist, deutlich zu erkennen. Die Existenz der Pentagon-Muster korreliert mit dem offensichtlich steuernden und die Symmetrie bestimmenden Einfluß der zentralen pentagonalen $\mathrm{MoO}_{7^{-}}$ Bipyramide („nullte Generation“), mit der fünf $\mathrm{MoO}_{6}$ Oktaeder der „ersten Generation“ verknüpft sind. Die fünf $\mathrm{MoO}_{6}$-Oktaeder der ,zweiten Generation“ sind dann mit denen der ersten über Ecken verknüpft, wobei die der zweiten mit weiteren $\mathrm{MoO}_{6}$-Polyedern die angrenzenden $\mathrm{Mo}^{\mathrm{V}}-\mathrm{Mo}^{\mathrm{V}_{-}}$ Hanteln der relativ stabilen $\mathrm{Mo}_{2}^{\mathrm{V}} \mathrm{O}_{4}{ }^{2+}$-Gruppe bilden.

In diesem Zusammenhang ist sicherlich auffallend, daß man einerseits mit den $\left\{\mathrm{Mo}_{11}\right\} \equiv\left\{(\mathrm{Mo})^{0}\left(\mathrm{Mo}_{5}\right)^{\mathrm{I}}\left(\mathrm{Mo}_{5}\right)^{\mathrm{II}}\right\}$-Einheiten (Motiven) nicht nur Riesenkugeln ,generieren“ kann, sondern daß $\left\{\mathrm{Mo}_{11}\right\}$-Einheiten auch das Basismotiv für verschiedene riesenradförmige Polyoxomolybdatstrukturen

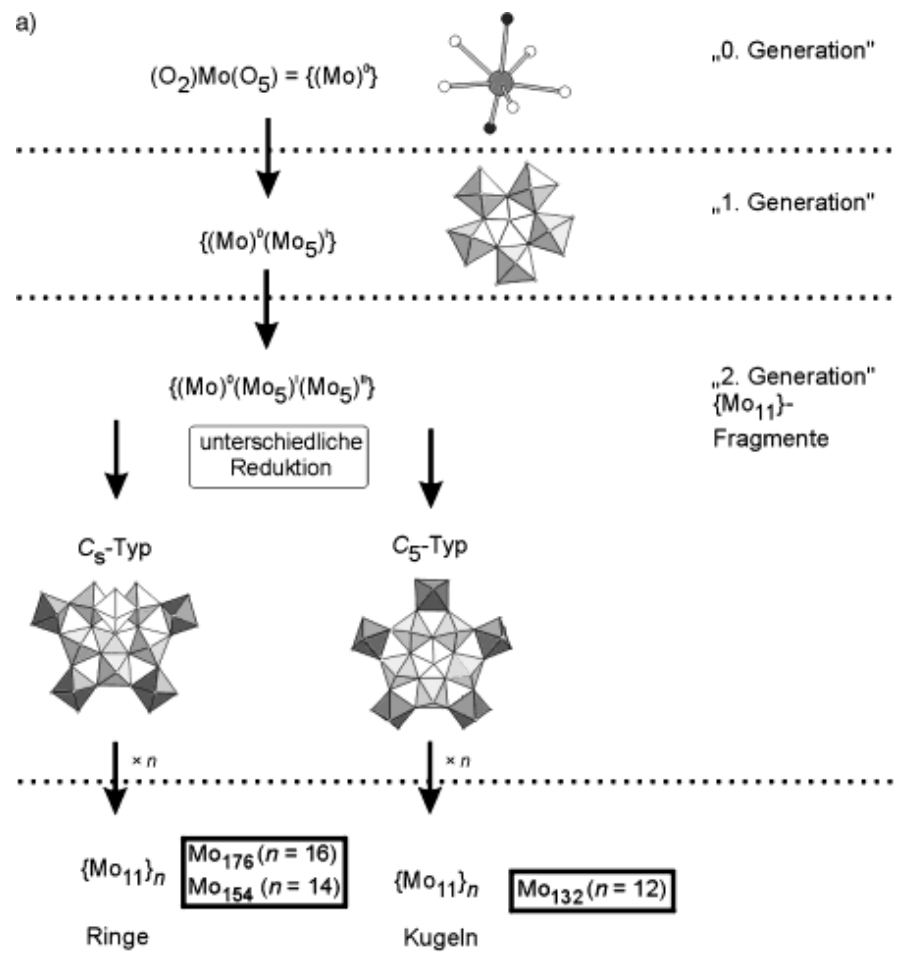

b)

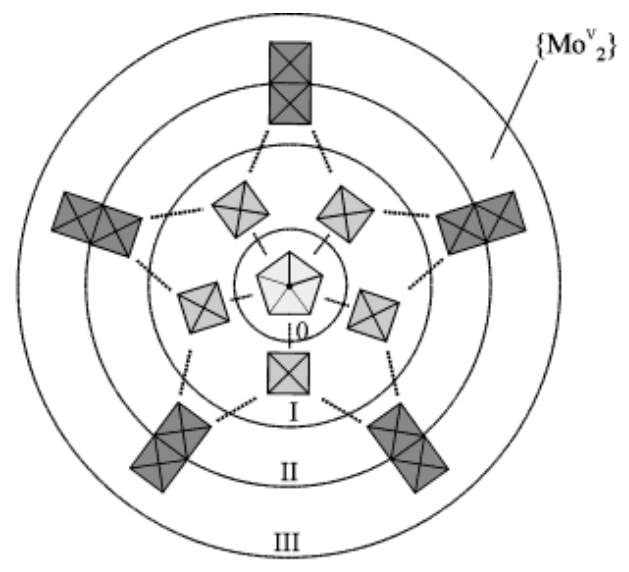

$\left\{(\mathrm{Mo})^{0}\left(\mathrm{Mo}_{5}\right)^{1}\left(\mathrm{Mo}_{5}\right)^{\mathrm{I}}-\left(\mathrm{Mo}_{5}\right)^{\mathrm{In}}\right\}$

Abbildung 2. a) Schematische (formale) Beziehungen zwischen Basisstrukturelementen und Motiven molekularer Riesenkugeln und Riesenräder sowie b) der formale Bezug zum $\left\{(\mathrm{Mo})^{0}\left(\mathrm{Mo}_{5}\right)^{\mathrm{I}}\left(\mathrm{Mo}_{5}\right)^{\mathrm{II}}-\left(\mathrm{Mo}_{5}\right)^{\mathrm{II}}\right\} \equiv$ $\left\{\mathrm{Mo}_{16}\right\}$-Motiv (,Deckel“ der Riesenkugel).

der Art $\left\{\mathrm{Mo}_{11}\right\}_{n}(n=14,16)$ darstellen (Abbildung 2 und 3). Nur im Falle stärkerer Reduktion sind - wie im vorliegenden Fall - die $\left\{\left(\mathrm{Mo}_{5}\right)^{\amalg}\right\}$-Zentren äquivalent, was dann letztlich eine Anordnung mit $C_{5}$-Symmetrie ermöglicht. (Zur Bildung des kugelförmigen Gebildes sind dabei allerdings die oben genannten zweizähnigen Liganden erforderlich, wobei sich die Kugelstrukturen formal durch Verknüpfung von 12 dieser regulären $\left\{\mathrm{Mo}_{11}\right\}$-Pentagone ergeben.) Bei geringerem Reduktionsgrad und in Abwesenheit des Liganden resultiert die Verschiebung eines $\mathrm{MoO}_{6}$-Oktaeders und damit eine nieder- 

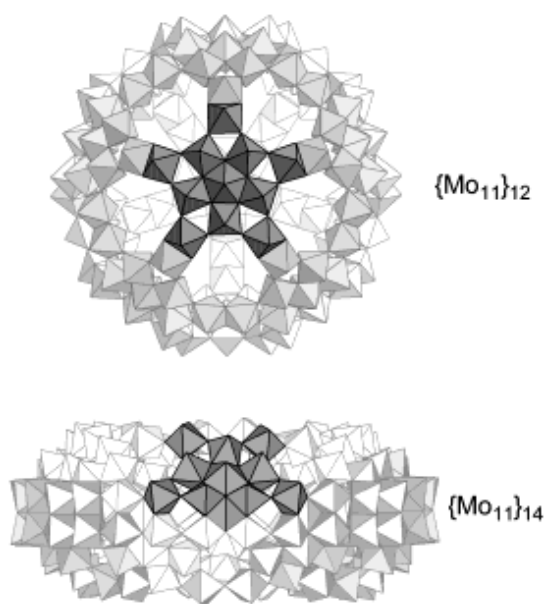

Abbildung 3. Strukturvergleich der ring- und kugelförmigen Cluster $\left\{\mathrm{Mo}_{11}\right\}_{14}$ bzw. $\left\{\mathrm{Mo}_{11}\right\}_{12}$ mit hervorgehobenen $\left\{\mathrm{Mo}_{11}\right\}$-Einheiten.

symmetrische $C_{\mathrm{s}}-\left\{\mathrm{Mo}_{11}\right\}$-Einheit (Abbildung $2 \mathrm{a}$ und 3), die in den Ringen vom $\left\{\mathrm{Mo}_{154}\right\}$ - und $\left\{\mathrm{Mo}_{176}\right\}-\mathrm{Typ}^{[8,9]}$ vorkommt $\left(\left(\mathrm{Mo}_{11}\right)_{14}\right.$ bzw. $\left.\left(\mathrm{Mo}_{11}\right)_{16}\right)$. Hier sollte allerdings nochmals betont werden, daß diese Betrachtungsweise nicht direkt mit dem Bildungsvorgang korreliert, allerdings für eine strukturchemische Betrachtungsweise sehr nützlich erscheint.

Wir meinen, daß die hier mitgeteilten Ergebnisse den Weg $\mathrm{zu}$ einer neuen Art von Supramolekularer Nanochemie aufzeigen. Die synthetisierten „Gefäße“ erlauben nämlich den Einschluß und darüber hinaus auch den gemeinsamen (!) Transport verschiedener Molekül- und/oder Ionenkollektive. Da der hier beschriebene Austausch der Acetatliganden mit vielen anderen Liganden/Gästen, z.B. auch solchen mit größeren organischen Resten, realisierbar ist, können im Kugelinneren auch neue, maßgeschneiderte Oberflächenstrukturen vor allem mit amphiphilen Liganden gezielt konstruiert werden. Überdies kann durch Steuerung der Reaktionsbedingungen der Grad der Substitution des Acetatliganden, z. B. durch nicht zu stark koordinierende Liganden, quasi variiert werden. Mit einem kleineren Liganden als dem in $\mathbf{2 a}$ (z. B. $\mathrm{HCOO}^{-}$statt $\mathrm{CH}_{3} \mathrm{COO}^{-}$) ist es sogar möglich, zwiebelförmige Anordnungen von eingeschlossenen van-derWaals-Clustern, z. B. eines $\left(\mathrm{H}_{2} \mathrm{O}\right)_{n}$-Kollektivs oder -Clusters, zu generieren. ${ }^{[7]}$ In diesem Falle beeinflußt nur die kugelförmige Clusterschale die Art der Organisation des zentralen Gästekollektivs im Hohlraum des Keplerat-Clusters. Weiterhin gibt es die Option, durch die 20 Öffnungen von 2 a mit einem Durchmesser von ca. $0.35 \mathrm{~nm}$, die durch die $\left\{\mathrm{Mo}_{9} \mathrm{O}_{9}\right\}$ Ringe begrenzt werden, kleinere Gäste wie kleine organische Moleküle einzuschleusen. Entsprechende Untersuchungen sind im Gange. Auf jeden Fall ist im Bereich der Polyoxometallat-Chemie noch viel Aufregendes (siehe auch Lit. $[8,9])$ und sicher auch eine (topologisch) vergleichbare Vielfalt wie bei den Fullerenen ${ }^{[10]}$ zu erwarten.

\section{Experimentelles}

1: Eine Lösung von $\mathrm{Na}_{2} \mathrm{MoO}_{4} \cdot 2 \mathrm{H}_{2} \mathrm{O}(7.67 \mathrm{~g}, 31.7 \mathrm{mmol})$ und $\mathrm{CH}_{3} \mathrm{COO}$ $\mathrm{Na} \cdot 3 \mathrm{H}_{2} \mathrm{O}(12.50 \mathrm{~g}, 91.9 \mathrm{mmol})$ in $250 \mathrm{~mL} \mathrm{H} \mathrm{H}_{2} \mathrm{O}$ wird unter Rühren mit $\mathrm{N}_{2} \mathrm{H}_{4} \cdot \mathrm{H}_{2} \mathrm{SO}_{4}(0.80 \mathrm{~g}, 6.2 \mathrm{mmol})$ und $65 \mathrm{~mL}$ 50proz. $\mathrm{CH}_{3} \mathrm{COOH}$ versetzt und anschließend 10 min weitergerührt (Farbwechsel allmählich nach Grün, während $\mathrm{N}_{2} \mathrm{H}_{4} \cdot \mathrm{H}_{2} \mathrm{SO}_{4}$ langsam abreagiert; $\mathrm{pH}$-Wert: ca. 4.0). Nach Zugabe von $\mathrm{NaCl}(5.00 \mathrm{~g}, 85.6 \mathrm{mmol})$ läßt man die Reaktionslösung in einem offenen Becherglas ( $400 \mathrm{~mL}$, weite Form) drei bis vier Wochen im Abzug stehen, wobei das Volumen der Lösung auf ca. $50 \mathrm{~mL}$ reduziert wird. Hierbei zersetzt sich offensichtlich das zunächst entstehende 2a durch partielle Oxidation, wobei innerhalb der ersten Stunden ein langsamer Farbwechsel der Lösung nach Dunkelbraun bei gleichzeitigem Auftreten einer Gasentwicklung beobachtet wird. Beim Eindunsten fallen mehrere Fraktionen aus: zuerst ein dunkelbrauner Niederschlag (Abfiltration bei einem Flüssigkeitsvolumen von ca. $150 \mathrm{~mL}$ ), dann orangebraune Kristalle von $\mathrm{Na}_{10}\left[\mathrm{H}_{4} \mathrm{Mo}_{10}^{\mathrm{VI}} \mathrm{Mo}_{8}^{\mathrm{V}} \mathrm{O}_{56}\left(\mathrm{CH}_{3} \mathrm{COO}\right)_{2}\right] \cdot$ ca. $34 \mathrm{H}_{2} \mathrm{O} \cdot 3 \mathrm{CH}_{3} \mathrm{COO}$ $\mathrm{Na}^{[11]}$ und ein Kristallgemisch aus farblosen Kristallen und schwarzbraunen, säulenförmigen Kristallen von 1. Zur Isolierung wird das Kristallgemisch über eine D2-Glasfritte von der öligen, tiefbraunen Mutterlauge abgesaugt, mehrfach mit einer wäßrigen NatriumacetatPufferlösung (siehe unten) sowie anschließend mit 2-Propanol gewaschen und über $\mathrm{CaCl}_{2}$ getrocknet. Die einzelnen Kristallfraktionen können nur mechanisch voneinander getrennt werden. Gesamtausbeute: ca. $3.5 \mathrm{~g}$ (ca. $35 \%$ 1, ca. $15 \% \mathrm{Na}_{10}\left[\mathrm{H}_{4} \mathrm{Mo}_{18} \mathrm{O}_{56}\left(\mathrm{CH}_{3} \mathrm{COO}\right)_{2}\right] \cdot$ ca. $34 \mathrm{H}_{2} \mathrm{O} \cdot 3 \mathrm{CH}_{3} \mathrm{COONa}$ und ca. $50 \%$ farblose Kristalle). 1 ist sehr gut löslich in $\mathrm{H}_{2} \mathrm{O}$. Charakteristische IR-Banden (KBr-Preßling; 1800-500 $\left.\mathrm{cm}^{-1}\right): \tilde{v}\left[\mathrm{~cm}^{-1}\right]=1628(\mathrm{~m}$, $\left.\delta\left(\mathrm{H}_{2} \mathrm{O}\right)\right), 1543\left(\mathrm{~s}, v_{\mathrm{as}}(\mathrm{COO})\right), 1447(\mathrm{~m}), 1412(\mathrm{~m})\left(\delta\left(\mathrm{CH}_{3}\right), \tilde{v}_{\mathrm{s}}(\mathrm{COO})\right), 971(\mathrm{~s})$, $937(\mathrm{w}-\mathrm{m})\left(v_{\mathrm{as}}(\mathrm{Mo}=\mathrm{O})\right), 854(\mathrm{~s}), 795(\mathrm{vs}), 724(\mathrm{~s}), 627(\mathrm{w}), 566(\mathrm{~s})$; charakteristische Raman-Banden (Festkörper und Lösung; $\lambda_{\mathrm{e}}=1064 \mathrm{~nm} ; 1000$ $\left.200 \mathrm{~cm}^{-1}\right): \tilde{v}\left[\mathrm{~cm}^{-1}\right]: 953(\mathrm{w}-\mathrm{m}), 934(\mathrm{w}-\mathrm{m})(\mathrm{v}(\mathrm{Mo}=\mathrm{O})), 874(\mathrm{~s}), \mathrm{ca} .845(\mathrm{sh})$, 371(m), 313(w-m); UV/Vis (Festkörperreflexionsspektrum/Verreibung auf Cellulose): $\lambda$ [nm]: ca. 440 (br), ca. 600 (br). Die spektroskopischen Daten sind nahezu identisch mit denen von 2 (gleiche Probentechnik wie in Lit. [2], dort irrtümlich anders angegeben).

Herstellung der Natriumacetat-Pufferlösung zum Waschen: $500 \mathrm{~mL}$ einer 50proz. wäßrigen $\mathrm{CH}_{3} \mathrm{COOH}$-Lösung werden unter Kühlung und Rühren mit einer 20proz. wäßrigen Natronlauge auf einen $\mathrm{pH}-$ Wert von 4.0 bis 4.2 eingestellt.

3: Eine Lösung von $\mathbf{2}^{[2]}(1.0 \mathrm{~g}, 0.04 \mathrm{mmol})$ in $50 \mathrm{~mL} \mathrm{H} \mathrm{H}_{2} \mathrm{O}$ wird unter Rühren mit $\mathrm{NaH}_{2} \mathrm{PO}_{2} \cdot \mathrm{H}_{2} \mathrm{O}(1.0 \mathrm{~g}, 9.43 \mathrm{mmol})$ und ca. $0.8 \mathrm{~mL}$ 50proz. $\mathrm{H}_{3} \mathrm{PO}_{2}$ versetzt (pH-Wert: 2.0) und $24 \mathrm{~h}$ bei einer Temperatur von $20^{\circ} \mathrm{C}$ gerührt. Nach anschließender Zugabe von $\mathrm{NH}_{4} \mathrm{Cl}(2.0 \mathrm{~g}, 37.4 \mathrm{mmol})$ und Abkühlung auf $15^{\circ} \mathrm{C}$ fallen aus der dunkelbraunen Lösung innerhalb von $4 \mathrm{~d}$ langsam dunkelbraune, rhomboederförmige Kristalle von $\mathbf{3}$ aus, die über eine Glasfritte abgesaugt und an der Luft getrocknet werden. Ausbeute: $0.55 \mathrm{~g}$ ( $56 \%$ bezogen auf eingesetztes 2). Charakteristische IR-Banden (KBr-Preßling; $\left.1800-500 \mathrm{~cm}^{-1}\right): \tilde{v}\left[\mathrm{~cm}^{-1}\right]=1620\left(\mathrm{~m}, \delta\left(\mathrm{H}_{2} \mathrm{O}\right)\right)$, ca. 1425 $(\mathrm{sh}), 1402(\mathrm{~s})\left(\delta_{\text {as }}\left(\mathrm{NH}_{4}^{+}\right)\right), 1118(\mathrm{~m}), 1075(\mathrm{w})$, ca. $1033(\mathrm{w})\left(\mathrm{H}_{2} \mathrm{PO}_{2}\right), 973$ (s), ca. $940(\mathrm{w})(v(\mathrm{Mo}=\mathrm{O})), 857$ (s), 801 (s), 724 (vs), 630 (m), 572 (s); charakteristische Raman-Banden (Festkörper und Lösung; $\lambda_{\mathrm{e}}=1064 \mathrm{~nm}$; $\left.1000-200 \mathrm{~cm}^{-1}\right): \tilde{v}\left[\mathrm{~cm}^{-1}\right]=939(\mathrm{~m}, v(\mathrm{Mo}=\mathrm{O})), 881(\mathrm{~s}), \mathrm{ca} .850(\mathrm{sh}), 374$ (m), $304(\mathrm{~m})$; UV/Vis (Festkörperreflexionsspektrum/Verreibung auf Cellulose): $\lambda[\mathrm{nm}]=$ ca. 450 (br), ca. 600 (br).

4: Eine Lösung von $2^{[2]}(0.50 \mathrm{~g}, 0.018 \mathrm{mmol})$ in $25 \mathrm{~mL} \mathrm{H}_{2} \mathrm{O}$ wird unter Rühren mit $\mathrm{Na}_{2} \mathrm{SO}_{4}(0.35 \mathrm{~g}, 2.5 \mathrm{mmol})$ und ca. $1 \mathrm{~mL} 15$ proz. Salzsäure versetzt. Man erwärmt die Reaktionslösung (pH-Wert: ca. 4.5) unter Rühren $48 \mathrm{~h}$ im Ölbad auf $30^{\circ} \mathrm{C}$. (Die für die Röntgenstrukturanalyse verwendeten Kristalle wurden in geeigneter Qualität dadurch erhalten, daß noch vor der Zugabe von $\mathrm{NH}_{4} \mathrm{Cl}$ kurz auf $90^{\circ} \mathrm{C}$ erhitzt wurde.) Nach Zugabe von $\mathrm{NH}_{4} \mathrm{Cl}(0.4 \mathrm{~g}, 7.5 \mathrm{mmol})$ fallen nach Abkühlen auf $18^{\circ} \mathrm{C}$ aus der dunkelbraunen Lösung innerhalb von $4 \mathrm{~d}$ langsam dunkelbraune, rhomboedrische Kristalle von $\mathbf{4}$ aus, die über eine Glasfritte abgesaugt und an der Luft getrocknet werden. Ausbeute: $0.2 \mathrm{~g}$ (40\% bezogen auf eingesetztes 2). 4 ist gut löslich in $\mathrm{H}_{2} \mathrm{O}$ und mäßig löslich in EtOH. Charakteristische IR-Banden (KBr-Preßling; $\left.1800-500 \mathrm{~cm}^{-1}\right): \tilde{v}\left[\mathrm{~cm}^{-1}\right]=$ $1625\left(\mathrm{~m}, \delta\left(\mathrm{H}_{2} \mathrm{O}\right)\right), 1540\left(\mathrm{w}, v_{\text {as }}(\mathrm{COO})\right)$, ca. $1425(\mathrm{sh}), 1401(\mathrm{~s})\left(\delta_{\text {as }}\left(\mathrm{NH}_{4}^{+}\right)\right)$, $1187(\mathrm{~m}), 1132(\mathrm{~m}), 1044(\mathrm{w}-\mathrm{m})\left(\mathrm{v}_{\mathrm{as}}\left(\mathrm{SO}_{3}\right)\right), 970(\mathrm{~s}), 936(\mathrm{w}-\mathrm{m})(\mathrm{v}(\mathrm{Mo}=\mathrm{O}))$, $855(\mathrm{~s}), 798(\mathrm{~s}), 726(\mathrm{~m}), 632(\mathrm{w}-\mathrm{m}), 572(\mathrm{~m})$; charakteristische RamanBanden (Festkörper und Lösung; $\lambda_{\mathrm{e}}=1064 \mathrm{~nm} ; 1000-200 \mathrm{~cm}^{-1}$ ): $\tilde{v}\left[\mathrm{~cm}^{-1}\right]=942(\mathrm{~m})(\mathrm{v}(\mathrm{Mo}=\mathrm{O})), 879(\mathrm{~s}), \mathrm{ca} .845(\mathrm{sh}), 375(\mathrm{~m}), 312(\mathrm{w}-\mathrm{m})$; UV/Vis (Festkörperreflexionsspektrum/Verreibung auf Cellulose): $\lambda[\mathrm{nm}]=$ ca. 450 (br), ca. 600 (br).

Eingegangen am 26. März 1999 [Z13215] International Edition: Angew. Chem. Int. Ed. 1999, 38, 3241-3245 
Stichwörter: Cluster $\cdot$ Käfigverbindungen $\cdot$ Polyoxometallate · Strukturaufklärung - Supramolekulare Chemie

[1] Der Titel dieser Arbeit bezieht sich auf einen Beitrag von P. C. H. Mitchell („Open and shut case for anions“, Nature 1990, 348, 15-16; Rubrik: News and Views), der sich wiederum auf eine frühere Publikation von uns über eine entsprechende Wirt-Gast-Chemie bezog (A. Müller, M. Penk, R. Rohlfing, E. Krickemeyer, J. Döring, Angew. Chem. 1990, 102, 927-929; Angew. Chem. Int. Ed. Engl. 1990, 29, 926-927). Der genannte Titel ist nach unserer Meinung besonders jetzt durch die vorliegende Zuschrift in vollem Umfange gerechtfertigt, da in unserer damaligen Arbeit im Prinzip nur geschlossene Wirte behandelt wurden. Auch auf die Riesenräder läßt sich der Begriff „Open and Shut" beziehen (A. Müller, S. Q. N. Shah, H. Bögge, M. Schmidtmann, Nature 1999, 397, 48-50).

[2] a) A. Müller, E. Krickemeyer, H. Bögge, M. Schmidtmann, F. Peters, Angew. Chem. 1998, 110, 3567-3571; Angew. Chem. Int. Ed. 1998, 37, 3360-3363; b) siehe auch: A. Müller, S. Sarkar, S. Q. N. Shah, H. Bögge, M. Schmidtmann, Sh. Sarkar, P. Kögerler, B. Hauptfleisch, A. X. Trautwein, V. Schünemann, Angew. Chem. 1999, 111, $3435-$ 3439; Angew. Chem. Int. Ed. 1999, 38, $3238-3241$ (voranstehende Zuschrift).

[3] Die Pythagoräer glaubten, daß sich die Ordnung der Welt, ihre Harmonie und Symmetrieeigenschaften durch Zahlenverhältnisse festlegen lassen: Aristoteles schrieb in seiner Metaphysik, daß nach den Pythagoräern (allerdings entgegen seiner Auffassung) die Elemente der Zahlen die Elemente aller Dinge seien und die verschiedenen Strukturtypen sich durch verschiedene Zahlen unterscheiden lassen (vgl.: A. Stückelberger, Einführung in die antiken Naturwissenschaften, Wiss. Buchgesellschaft, Darmstadt, 1988, S. 12).

[4] Das Ausmaß des Austausches des Acetatliganden hängt von der Nucleophilie des zugesetzten Liganden und dem pH-Wert der Lösung ab. Der Austausch verläuft z. B. beim Phosphat- und Hypophosphition (zumindest bei niedrigem pH-Wert) bereits bei Raumtemperatur vollständig, beim Sulfation jedoch nur partiell. Über das PhosphatKeplerat sollen hier keine Details mitgeteilt werden, da unsere bisherigen Untersuchungen auf einen interessanten Befund hindeuten, der separat erörtert werden müßte, nämlich, daß man z. B. durch Änderung des $\mathrm{pH}-$ Wertes die relative Aufnahme von $\mathrm{HPO}_{4}{ }^{2-}$ - und $\mathrm{H}_{2} \mathrm{PO}_{4}^{-}$-Ionen steuern kann.

[5] 1: Raumgruppe $P m n 2_{1} ; a=49.117(4), b=34.898(3), c=25.223(2) \AA$, $V=43234(7) \AA^{3} ; Z=2 ; 207015$ Reflexe erhalten bei $183 \mathrm{~K}$ aus 1271 Aufnahmen, die jeweils $0.3^{\circ}$ in $\omega$ abdecken $\left(2 \theta_{\max }=50^{\circ}\right)$; Strukturlösung mit Direkten Methoden, $R=0.0896$ für 35329 unabhängige Reflexe $\left(F_{\mathrm{o}}>4 \sigma\left(F_{\mathrm{o}}\right)\right)$. 3: Raumgruppe $R \overline{3} ; \quad a=32.719(1), \quad c=$ 73.567(2) $\AA, V=68205(3) \AA^{3} ; Z=3 ; 134487$ Reflexe erhalten bei $183 \mathrm{~K}$ aus 1271 Aufnahmen, die jeweils $0.3^{\circ}$ in $\omega$ abdecken $\left(2 \theta_{\max }=\right.$ $54^{\circ}$ ). Strukturlösung mit Direkten Methoden, $R=0.0457$ für 24569 unabhängige Reflexe $\left(F_{\mathrm{o}}>4 \sigma\left(F_{\mathrm{o}}\right)\right)$. 4: Raumgruppe $R \overline{3} ; a=32.695(1)$, $c=73.529(3) \AA, V=68069(3) \AA^{3} ; Z=3 ; 134980$ Reflexe erhalten bei
$183 \mathrm{~K}$ aus 1271 Aufnahmen, die jeweils $0.3^{\circ}$ in $\omega$ abdecken $\left(2 \theta_{\max }=\right.$ $54^{\circ}$ ). Strukturlösung mit Direkten Methoden, $R=0.0409$ für 23684 unabhängige Reflexe $\left(F_{\mathrm{o}}>4 \sigma\left(F_{\mathrm{o}}\right)\right)$. Die vermessenen Kristalle wurden direkt aus den jeweiligen Reaktionslösungen entnommen und sofort mit einem Bruker-axs-SMART-Diffraktometer vermessen, um einen Kristallwasserverlust zu vermeiden, wobei die Messungen mehrerer individueller Kristalle jeweils zum gleichen Ergebnis führten. Die Strukturen wurden mit dem Programm SHELXS-97 gelöst, mit den Programmen SHELXL-93 bzw. SHELXL-97 verfeinert, und Absorptionskorrekturen erfolgten mit dem Programm SADABS (G. M. Sheldrick, Universität Göttingen, 1997 und 1993). Es gab Fehlordnungsprobleme bei folgenden Einheiten: 1) bei allen kleineren Gitterbestandteilen, 2) Acetatliganden auf verschiedenen Positionen bei $\mathbf{1}$ und 4, 3) bei terminalen MoO-Gruppen mit zugehörigen trans-ständigen $\mathrm{H}_{2} \mathrm{O}$-Liganden bei 4 , 4) bei $\mathrm{H}_{2} \mathrm{PO}_{2}^{-}$Liganden bei 3 und $\mathrm{HSO}_{4}{ }^{-}$-Liganden bei 4. Aus (1) und (4) resultieren für die Formeln übliche Fehlerbreiten. Die Strukturgraphiken wurden mit dem Programm DIAMOND 2.1 (K. Brandenburg, Crystal Impact GbR, 1999) angefertigt. Weitere Einzelheiten zur Kristallstrukturuntersuchung von $\mathbf{3}$ können beim Fachinformationszentrum Karlsruhe, D-76344 Eggenstein-Leopoldshafen (Fax: (+49)7247-808-666; E-mail: crysdata@fiz-karlsruhe.de), unter der Hinterlegungsnummer CSD-410885 angefordert werden. Die kristallographischen Daten (ohne Strukturfaktoren) der in dieser Veröffentlichung beschriebenen Strukturen von $\mathbf{1}$ und $\mathbf{4}$ wurden als ,supplementary publication no.“ CCDC-132028 bzw. -132029 beim Cambridge Crystallographic Data Centre hinterlegt. Kopien der Daten können kostenlos bei folgender Adresse in Großbritannien angefordert werden: CCDC, 12 Union Road, Cambridge CB21EZ (Fax: (+44)1223-336-033; E-mail: deposit@ccdc.cam.ac.uk).

[6] I. D. Brown in Structure and Bonding in Crystals, Vol. II (Hrsg.: M. O'Keeffe, A. Navrotsky), Academic Press, New York, 1981, S. 1-30.

[7] A. Müller, V. P. Fedin, C. Kuhlmann, H. Bögge, M. Schmidtmann, Chem. Commun. 1999, 927-928.

[8] a) A. Müller, F. Peters, M. T. Pope, D. Gatteschi, Chem. Rev. 1998, 98, $239-271$; b) A. Müller, E. Krickemeyer, H. Bögge, M. Schmidtmann, C. Beugholt, S. K. Das, F. Peters, Chem. Eur. J. 1999, 5, 1496-1502; c) A. Müller, P. Kögerler, C. Kuhlmann, Chem. Commun. 1999, 1347 1358.

[9] a) M. T. Pope, Heteropoly and Isopoly Oxometalates, Springer, Berlin, 1983; b) Polyoxometalates: From Platonic Solids to Anti-Retroviral Activity (Hrsg.: M. T. Pope, A. Müller), Kluwer, Dordrecht, 1994.

[10] a) H. W. Kroto, J. R. Heath, S. C. O'Brien, R. F. Curl, R. E. Smalley, Nature 1985, 318, 162-163; b) Buckminsterfullerenes (Hrsg.: W. E. Billups, M. A. Ciufolini), VCH, Weinheim, 1993; c) Von Fuller bis zu Fullerenen: Beispiele einer interdisziplinären Forschung (Hrsg.: W. Krätschmer, H. Schuster), Vieweg, Braunschweig, 1996

[11] Raumgruppe $P \overline{1} ; a=12.1293(5), b=15.2523(6), c=15.2852(6) \AA$, $\alpha=114.911(1), \beta=94.227(1), \gamma=101.647(1)^{\circ}, V=2471.4(2) \AA^{3} ; R=$ 0.0358 für 12491 unabhängige Reflexe $\left(F_{\mathrm{o}}>4 \sigma\left(F_{\mathrm{o}}\right)\right)$. 